\title{
Optimizing the Dosage Regimen of Micafungin against Candida spp in HIV Positive Patients with EC Based on Mote Carlo Simulation
}

\author{
Ying Wang1, Jingyi Zhao², Song Jiang1, Yinhui Yao ${ }^{1}$, Hainan Wen ${ }^{3}$ \\ ${ }^{1}$ Department of Pharmacy, The Affiliated Hospital of Chengde Medical College, Chengde, China \\ ${ }^{2}$ Department of Functional Center, Chengde Medical College, Chengde, China \\ ${ }^{3}$ Department of Clinical Laboratory, The Affiliated Hospital of Chengde Medical College, Chengde, China \\ Email: *yaoyh_gc@163.com
}

How to cite this paper: Wang, Y., Zhao, J.Y., Jiang, S., Yao, Y.H. and Wen, H.N. (2020) Optimizing the Dosage Regimen of Micafungin against Candida spp in HIV Positive Patients with EC Based on Mote Carlo Simulation. Open Journal of Preventive Medicine, 10, 120-125.

https://doi.org/10.4236/ojpm.2020.106009

Received: May 19, 2020

Accepted: June 14, 2020

Published: June 17, 2020

Copyright $\odot 2020$ by author(s) and Scientific Research Publishing Inc. This work is licensed under the Creative Commons Attribution International License (CC BY 4.0).

http://creativecommons.org/licenses/by/4.0/

\begin{abstract}
The objective of our study was to explore the possibility of the antifungal efficacy of various micafungin dosage regimens against Candida spp in HIV positive patients with EC. According to pharmacokinetic/pharmacodynamics parameters of micafungin in HIV positive patients and MICs distribution of micafungin against Candida spp. in published studies, the dosage regimens of micafungin were 50, 100 and $150 \mathrm{mg}$ QD iv. Monte Carlo Simulation analysed the probability of target attainment and cumulative fraction of response. The results showed that micafungin has good antifungal effect in treating HIV positive patients with EC when pathomycetes are Candida albicans, Candida glabrata or Candida tropicalis, in dosage at $100 \mathrm{mg}$ QD and $150 \mathrm{mg}$ QD.
\end{abstract}

\section{Keywords}

Monte Carlo Simulation, Micafungin, HIV Positive Patients, Esophageal Candidiasis

\section{Introduction}

In recent years, invasive fungal infections (IFIs) had been a significant factor in the morbidity and mortality of inpatients with invasive infections, especially in patients with immunodeficiency [1] [2]. The primary pathogenic fungi for IFIs are Candida albicans, Candida glabrata, Candida krusei, Candida parapsilosis and Candida tropicalis [1].

In patients with the human immunodeficiency virus (HIV) infection, eso- 
phagal candidiasis (EC) is a common and severe complication, the incidence is $15 \%-20 \%$, and micafungin has shown great efficacy and tolerability in treating EC in HIV positive patients [3]. Micafungin is an echinocandin antifungal agent, which plays an antifungal role in selective inhibiting the synthase of $\beta$-(1,3)-D-glucan in the fungal cell wall [4] [5]. Micafungin mainly binds to albumin in vivo, the protein binding rate in plasma is $99 \%$ [6]. In vivo, micafungin is metabolized by the liver and excreted through the biliary tract, and mainly excreted through faeces [7]. Studies showed that micafungin has antifungal activities against Candida spp. both in vitro and in vivo, even for fluconazole resistance fungus [8] [9] [10] [11].

Monte Carlo simulation (MCS) is a useful tool for clinical treatment of dose selection, which can be sufficient to evaluate the effect of antifungal drugs and minimise the possibility of antifungal drug resistance. MCS has been used to assess the dosing regimens of micafungin in morbidly obese patients [12], critically ill patients with invasive fungal infection [13], critically burned patients with abdominal disease [14] and children [15]. In this study, MCS was used to optimise the micafungin dosage regimen of EC in HIV positive patients, to provide a basis for clinical application.

\section{Materials and Methods}

\subsection{Pharmacokinetic Parameters}

Pharmacokinetic parameters for micafungin in HIV positive patients with EC from the literature [3], PK data of intravenous micafungin in HIV-positive patients are shown in Table 1. Micafungin is a concentration-dependent antifungal drug with long-term aftereffects, and the antifungal effect is measured by $f A U C_{24 h} / M I C$, PD target of Candida spp. is $f A U C_{24 h} / M I C=10$ [16], Free drug fraction $f$ is $1 \%$.

\subsection{The Minimum Inhibitory Concentration (MIC) Data}

The MICs distribution of Candida spp. is from the European Committee on Antimicrobial Susceptibility Testing (EUCAST) (http://www.eucast.org). The data has shown in Table 2.

\subsection{Monte Carlo Simulation}

The probability of target attainment (PTA) is the target value of Pharmacokinetic/pharmacodynamic (PK/PD); the calculation formula is

Table 1. Pharmacokinetic parameters for micafungin in HIV positive patients.

\begin{tabular}{cccc}
\hline PK parameters & $\mathbf{5 0} \mathbf{~ m g} /$ day $(\mathbf{n}=\mathbf{2 0})$ & $\mathbf{1 0 0} \mathbf{~} \mathbf{g} /$ day $(\mathbf{n}=\mathbf{2 0})$ & $\mathbf{1 5 0} \mathbf{~} \mathbf{g} /$ day $(\mathbf{n}=\mathbf{1 4})$ \\
\hline $\mathrm{CL}(\mathrm{mL} / \mathrm{h} / \mathrm{kg})$ & $19.3 \pm 5.9$ & $19.8 \pm 5.4$ & $20.4 \pm 5.5$ \\
$\mathrm{AUC}_{0-24}(\mathrm{~kg} \mathrm{~h} / \mathrm{mL})$ & $35.7 \pm 8.9$ & $74.5 \pm 18.7$ & $104.3 \pm 26.3$ \\
$\mathrm{C}_{\max }(\mu \mathrm{g} / \mathrm{mL})$ & $4.1 \pm 1.4$ & $8.0 \pm 2.4$ & $11.6 \pm 3.1$
\end{tabular}


Table 2. The MICs distribution of micafungin against Candida spp.

\begin{tabular}{|c|c|c|c|c|c|c|c|c|c|c|c|c|c|c|}
\hline \multirow{2}{*}{ Species } & \multirow{2}{*}{$\mathbf{n}$} & \multicolumn{13}{|c|}{$\mathrm{MIC}(\mu \mathrm{g} / \mathrm{ml})$} \\
\hline & & 0.002 & 0.004 & 0.008 & 0.016 & 0.032 & 0.064 & 0.125 & 0.25 & 0.5 & 1 & 2 & 4 & 8 \\
\hline Candida albicans & 1569 & 4 & 286 & 360 & 763 & 146 & 7 & 1 & 0 & 0 & 2 & 0 & 0 & 0 \\
\hline Candida glabrata & 692 & 0 & 90 & 182 & 273 & 135 & 3 & 5 & 0 & 1 & 1 & 2 & 0 & 0 \\
\hline Candida krusei & 483 & 0 & 1 & 0 & 4 & 26 & 185 & 215 & 37 & 9 & 2 & 4 & 0 & 0 \\
\hline Candida parapsilosis & 743 & 0 & 0 & 0 & 3 & 1 & 0 & 1 & 35 & 113 & 332 & 244 & 14 & 0 \\
\hline Candida tropicalis & 732 & 0 & 48 & 51 & 247 & 298 & 59 & 15 & 2 & 1 & 6 & 5 & 0 & 0 \\
\hline
\end{tabular}

$f A U C_{24 h} / M I C=(f \times$ dose $) /(C L \times M I C)$ MCS simulated ten thousand patients through Crystal Ball software (version 11.1.2.4.600, Oracle). $C L$ follows a logarithmic normal distribution, dose $(\mathrm{mg})$ and $\mathrm{f}$ follow a uniform distribution, and MICs follows the custom distribution.

Cumulative fraction of response (CFR) describe the expected probability of the target value of the corresponding strain population, the calculation formula is $C F R=\sum_{i=1}^{n} P T A i \times F i$. PTA $i$ is probability of target for the specific MIC; $F i$ is the probability of every MIC distribution for an individual fungal sample, to achieve an excellent antifungal effect, PTA > 90\% and CFR > 90\% [12] [13] [14] [15].

\section{Results}

\subsection{PTA Values}

PTA values of micafungin against Candida spp. in HIV positive patients under different MICs distribution shown in Figure 1. The results showed that in dosage at $50 \mathrm{mg}$, the 5 Candida spp can reach the target when MIC is less than $0.032 \mu \mathrm{g} / \mathrm{mL}$. In dosage at $100 \mathrm{mg}$ and $150 \mathrm{mg}$, the 5 Candida spp can attain the goal when MIC is less than $0.064 \mu \mathrm{g} / \mathrm{mL}$.

\subsection{CFR}

As the results are shown in Table 3, the effects of micafungin against Candida krusei and Candida parapsilosis are not sound, all CFR values cannot reach 90\% in every dosage. The results also showed a good effect in micafungin against Candida albicans and Candida glabrata, all CFR values are higher than $90 \%$. For Candida tropicalis, when the dosage of micafungin at $50 \mathrm{mg}$, CFR values below $90 \%$, when the dosage of micafungin at $150 \mathrm{mg}$ and $100 \mathrm{mg}$, the antifungal effect is proper, CRF values are higher than $90 \%$.

\section{Discussion}

Micafungin is one of three currently available echinocandins in the treatment of Candidiasis, and the FDA recommends a dose of $100 \mathrm{mg}$ QD for adult Candidiasis [17]. In HIV positive patients confirmed EC, no effect of race or gender 
Candida albicans

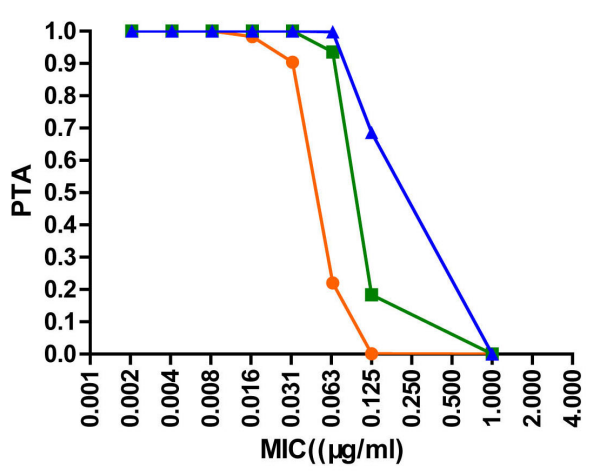

Candida tropicalis

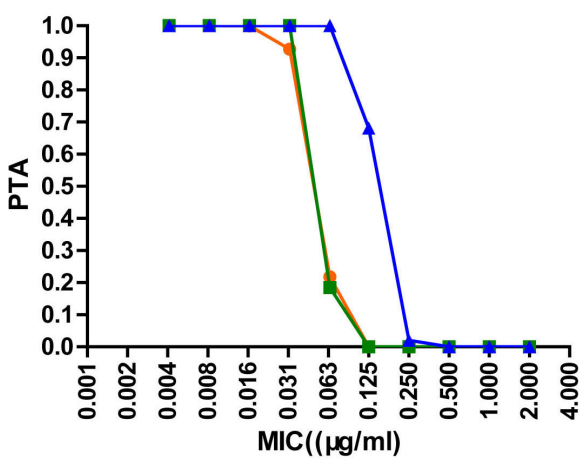

Candida krusei
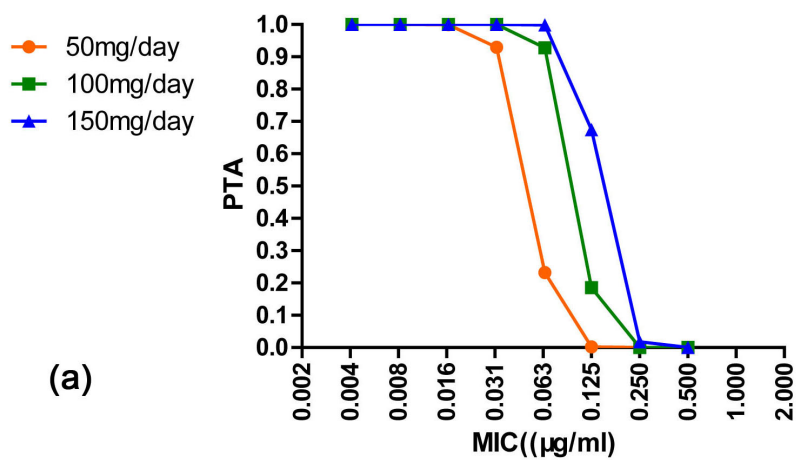

(b)

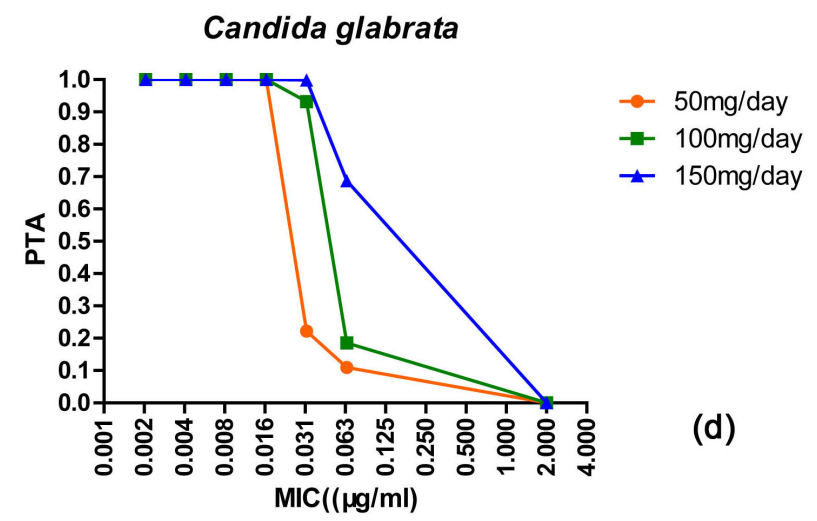

Candida parapsilosis
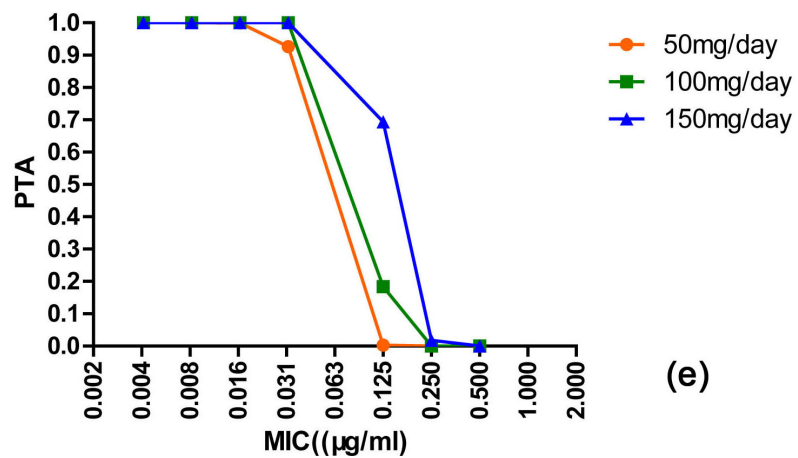

(e)

Figure 1. PTA of micafungin estimated at different dosage regimen against 5 Candida spp. in HIV positive patients with EC. (Key: 5 Candida spp. are Candida albicans, Candida glabrata, Candida krusei, Candida parapsilosis and Candida tropicalis. (a) to (e) show PTA values in of micafungin against 5 Candida spp. at different MICs in 3 dosage regimen: $50 \mathrm{mg} / \mathrm{qd}, 100 \mathrm{mg} / \mathrm{qd}$ and 150 mg/qd. (a) shows PTA values of Candida albicans, (b) shows PTA values of Candida krusei, (c) shows PTA values of Candida tropicalis, (d) shows PTA values of Candida glabrata, and (e) shows PTA values of Candida parapsilosis.)

Table 3. CFR (\%) of micafungin against Candida spp in HIV positive patients with EC.

\begin{tabular}{|c|c|c|c|}
\hline \multirow{2}{*}{ Species } & \multicolumn{3}{|c|}{ CFR\% } \\
\hline & $50 \mathrm{mg} /$ day & $100 \mathrm{mg} /$ day & $150 \mathrm{mg} /$ day \\
\hline Candida albicans & 97.75 & 99.79 & 99.85 \\
\hline Candida glabrata & 96.99 & 98.80 & 99.15 \\
\hline Candida krusei & 15.01 & 50.23 & 74.84 \\
\hline Candida parapsilosis & 0.53 & 0.57 & 0.71 \\
\hline Candida tropicalis & 86.75 & 95.89 & 97.40 \\
\hline
\end{tabular}


on the pharmacokinetics of micafungin [3].

Pharmacokinetics of micafungin in treating patients with EC was linear, predictable, which is similar to the published studies in healthy adults [3].

In our study, the antifungal effect of micafungin against different Candida spp in HIV positive patients with EC was quite different. For Candida krusei and Candida parapsilosis, micafungin has no antifungal impact, which is similar to the published studies in intensive care unit patients [18]. Our study also showed that micafungin against Candida albicans, Candida glabrata and Candida tropicalis has good antifungal effect in dosage greater than or equal to $100 \mathrm{mg}$, which is similar to FDA recommendation [17].

In this study, MCS used to carry out hypothesis analysis based on certain PK and strain data, which was beneficial to optimise the type and dosage of micafungin against Candida spp in HIV positive patients with EC. However, the research results of this paper also have some limitations, such as the MIC distribution of micafungin from some regions but not world-wide, so it can not reflect the development trend and change of the fungus in the future.

\section{Conclusion}

In summary, MCS is a simple, safe method to optimise dosage regimen according to the characteristics of fungi and PK/PD parameters. When pathomycetes are Candida albicans, Candida glabrata or Candida tropicalis, micafungin has good antifungal effect in HIV positive patients with EC, when pathomycetes are Candida krusei or Candida parapsilosis, other antifungal treatments are needed.

\section{Funding}

This study was funded by the Study and Development Fund for Sciences and Technology in Chengde City (No. 201701A086).

\section{Conflicts of Interest}

The authors declare no conflicts of interest regarding the publication of this paper.

\section{References}

[1] Kyriakidis, I., Tragiannidis, A., Munchen, S., et al. (2017) Clinical Hepatotoxicity Associated with Antifungal Agents. Expert Opinion on Drug Safety, 16, 149-165.

[2] Enoch, D.A., Yang, H.N., Aliyu, S.H., et al. (2017) The Changing Epidemiology of Invasive Fungal Infections. Methods in Molecular Biology, 1508, 17-65. https://doi.org/10.1007/978-1-4939-6515-1_2

[3] Under, N., Stevenson, P. and Baraldi, E. (2012) Pharmacokinetics of Micafungin in HIV Positive Patients with Confirmed Esophageal Candidiasis. European Journal of Drug Metabolism and Pharmacokinetics, 37, 31-38. https://doi.org/10.1007/s13318-011-0063-8

[4] Chen, S.C., Slavin, M.A. and Sorrell, T.C. (2011) Echinocandin Antifungal Drugs in Fungal Infections: A Comparison. Drugs, 71, 11-41. https://doi.org/10.2165/11585270-000000000-00000 
[5] Carver, P.L. (2004) Micafungin. The Annals of Pharmacotherapy, 38, 1707-1721. https://doi.org/10.1345/aph.1D301

[6] Muilwijk, E.W., Lempers, V.J.C., Burger, D.M., et al. (2015) Impact of Special Patient Populations on the Pharmacokinetics of Echinocandins. Expert Review of Anti-Infective Therapy, 13, 799-815. https://doi.org/10.1586/14787210.2015.1028366

[7] Wasmann, R.E., Muilwijk, E.W., Burger, D.M., et al. (2018) Clinical Pharmacokinetics and Pharmacodynamics of Micafungin. Clinical Pharmacokinetics, 57, 267-286. https://doi.org/10.1007/s40262-017-0578-5

[8] Maesaki, S., Hossain, M.A., Miyazaki, Y., et al. (2000) Efficacy of FK463, a (1,3)-beta-D-glucan Synthase Inhibitor, in Disseminated Azole-Resistant Candida Albicans Infection in Mice. Antimicrobial Agents and Chemotherapy, 44, 1728-1730. https://doi.org/10.1128/AAC.44.6.1728-1730.2000

[9] Messer, S.A., Diekema, D.J., Boyken, L., et al. (2006) Activities of Micafungin against 315 Invasive Clinical Isolates of Fluconazole-Resistant Candida spp. Journal of Clinical Microbiology, 44, 324-326. https://doi.org/10.1128/JCM.44.2.324-326.2006

[10] Pfaller, M.A., Boyken, L., Hollis, R.J., et al. (2008) In Vitro Susceptibility of Invasive Isolates of Candida spp. to Anidulafungin, Caspofungin, and Micafungin: Six Years of Global Surveillance. Journal of Clinical Microbiology, 46, 150-156. https://doi.org/10.1128/JCM.01901-07

[11] Warn, P.A., Sharp, A., Morrissey, G., et al. (2002) In Vivo Activity of Micafungin in a Persistently Neutropenic Murine Model of Disseminated Infection Caused by Candida tropicalis. Journal of Antimicrobial Chemotherapy, 50, 1071-1074. https://doi.org/10.1093/jac/dkf247

[12] Wasmann, R.E., Smit, C., Heine, R., et al. (2019) Pharmacokinetics and Probability of Target Attainment for Micafungin in Normal-Weight and Morbidly Obese Adults. Journal of Antimicrobial Chemotherapy, 74, 978-985. https://doi.org/10.1093/jac/dky554

[13] Boonstra, J.M., van der Elst, K.C., Veringa, A., et al. (2017) Pharmacokinetic Properties of Micafungin in Critically Ill Patients Diagnosed with Invasive Candidiasis. Antimicrobial Agents and Chemotherapy, 61, pii: e01398-17. https://doi.org/10.1128/AAC.01398-17

[14] García-de-Lorenzo, A., Luque, S., Grau, S., et al. (2016) Comparative Population Plasma and Tissue Pharmacokinetics of Micafungin in Critically Ill Patients with Severe Burn Injuries and Patients with Complicated Intra-Abdominal Infection. Antimicrobial Agents and Chemotherapy, 60, 5914-5921. https://doi.org/10.1128/AAC.00727-16

[15] Xu, G.Q., Zhu, L.Q., Ge, T.Y., et al. (2016) Optimal Micafungin Dosage Regimens in Pediatric Patients with Invasive Candida Infection. Chinese Journal of New Drugs and Clinical Remedies, 35, 482-486.

[16] Lepak, A.J. and Andes, D.R. (2015) Antifungal Pharmacokinetics and Pharmacodynamics. Cold Spring Harbor Perspectives in Medicine, 5, a019653. https://doi.org/10.1101/cshperspect.a019653

[17] Wasmann, R.E., Muilwijk, E.W., Burger, D.M., et al. (2018) Clinical Pharmacokinetics and Pharmacodynamics of Micafungin. Clinical Pharmacokinetics, 57, 267-286. https://doi.org/10.1007/s40262-017-0578-5

[18] Yang, Q.T., Wang, T.T., Xie, J., et al. (2016) Pharmacokinetic/Pharmacodynamic Adequacy of Echinocandins against Candida spp. in Intensive Care Unit Patients and General Patient Populations. International Journal of Antimicrobial Agents, 47, 397-402. https://doi.org/10.1016/j.ijantimicag.2016.02.004 